\title{
Estimulación de las funciones ejecutivas y su influencia en el rendimiento académico en escolares de primero básico
}

\section{Stimulation of the executive functions and their influence on academic perfor- mance of students of first grade of elementary school}

\begin{abstract}
Francisca Bernal-Ruiz ${ }^{1}$, Montserrat Rodríguez-Vera ${ }^{2}$ y Alonso Ortega ${ }^{3}$
${ }^{1}$ Psicóloga. Doctora en Psicología y Magíster en Pedagogía Universitaria. Docente e Investigadora de la Facultad de Educación de la Universidad de Playa Ancha de Ciencias de la Educación, Valparaíso, Chile. E-mail: fbernal@upla.cl

${ }^{2}$ Psicóloga. Máster en Neuropsicología y Magister en Psicología Social. Coordinadora Programa PACE, Universidad de Playa Ancha de Ciencias de la Educación, Chile.

E-mail: montserrat.rodriguez@upla.cl

${ }^{3}$ Psicólogo. Doctor en Ciencias y Magíster en Psicología. Docente de la Escuela de Fonoaudiología, Investigador del Centro de Investigación del Desarrollo en Cognición y Lenguaje (CIDCL) de la Universidad de Valparaíso, Chile. E-mail: alonso.ortega@uv.cl

Esta investigación fue financiada por el Convenio de Desempeño CD/PMI UPA 1203 de la Universidad de Playa Ancha y fue realizada en la Escuela Naciones Unidas D-245 de la comuna de Valparaíso, Chile.

Escuela D-245 Naciones Unidas de la Comuna de Valparaíso, Chile.
\end{abstract}

\section{Resumen}

El estudio de la estimulación de las funciones ejecutivas (FE) en la infancia ha generado un creciente interés investigativo en la última década, especialmente por su relación con el desempeño académico y éxito escolar. De acuerdo a esto, el presente estudio tiene por objetivo estudiar el efecto de un programa de estimulación de las FE sobre el desempeño ejecutivo y el rendimiento académico de una muestra de 43 estudiantes de primero básico de la comuna de Valparaíso (Chile), de edades entre 6 y 7 años. Para ello, se implementó un diseño factorial mixto. La intervención duró 12 semanas y se llevó a cabo en el aula, dirigida por un educador diferencial y con el apoyo de la profesora del curso. Los resultados evidencian la existencia de un efecto significativo del programa imple- mentado en la mejora del desempeño ejecutivo de los participantes, particularmente del grupo experimental. Tales efectos no fueron replicados en el rendimiento académico, lo que podría encontrar una posible explicación en los factores contextuales relacionados con las condiciones de precariedad de la institución educativa. Se observó además un efecto diferenciado del programa sobre el componente de control inhibitorio. Los hallazgos generales ponen de manifiesto la relevancia de la estimulación de las FE en la infancia temprana y los beneficios que esto puede reportar en contextos educativos. Además, sugiere el potencial aporte de la incorporación de este tipo de intervenciones en las prácticas docentes cotidianas.

Palabras clave: funciones ejecutivas; rendimiento académico; estimulación temprana; desarrollo cognitivo; diseño factorial mixto. 


\begin{abstract}
In the last decade, the study of executive functions has reached growing interest among researchers, especially because of its association with academic performance and school success. It is broadly accepted that the stimulation of executive functions during early childhood may predict and promote school success, mainly because of its impact on personal and academic skills. Therefore, early stimulation becomes critical to establish the optimal functionality of cognitive abilities, such as executive functions, for which the school context results appropriate. The development of executive functions has been associated with the maturation of prefrontal brain structures, and its early stimulation becomes especially relevant because it may both foster or constrain the children's individual capacities to learn and prosper along their lifetimes. Therefore, a better understanding of the executive function's development is critical for both monitoring and stimulating them, especially when the development of any of its dimensions shows some delay or maladjustment. The stimulation of children's executive performance may be well achieved through the implementation of intervention techniques, such as mindfulness, relaxation, strategical thinking, and psychomotor stimulation, among others. Accordingly, the present paper aims at studying the effect of an executive functions' stimulation program (EFSP) on both executive and academic performance. The EFSP stimulated three different brain pathways associated with prefrontal regions. The stimulated brain pathways were a) The fronto-striatal (i.e., cognitive circuit), b) The fronto-limbic (i.e., emotional), and c) The fronto-cerebellar (i.e., psychomotor circuit). The fronto-striatal pathway is involved in the ability to inhibit behaviors, and to attend when distractors appear, to favor the adequate use of the working memory and to allow the organization of plans and goals. The fronto-limbic pathway underlies the emotional and moti-
\end{abstract}

vational aspects of behavior, modulation the control of aggressive impulses. The fronto-cerebellar pathway provides the neural circuits which are necessary for the normal functioning of motor responses and time distribution, favoring the organization of behavior and the learning of habits. In our study, the intervention was conducted by a special education teacher, who was accompanied by the schoolteacher. The sample included fortythree first grade elementary school students of Valparaíso, Chile; aged between six and seven years old. A mixed factorial research design study was implemented, with a between-subjects factor defined by a two-level group variable (i.e., experimental vs. control) and a within-subject factor defined by a two-measures variable. Interactions between both factors were hypothesized and analyzed. Our results showed significant effects of the EFSP on the student's executive performance. Particularly, the experimental group showed better executive performance in comparison to their peers of the control group. Effect size measures were large according Cohen's (1988) criteria (i.e., partial eta squared; $\mathrm{h}_{\mathrm{p}}^{2}$ ). Such effects were not observed on academic performance, which could be explained by contextual factors related to the precarious conditions of the educational institution. An interesting result is the significant effect of the EFSP on the Inhibitory Control compound of the executive functions, which effect size can be also considered to be large. This finding may have an interesting impact on the implementation of programs intended to reinforce student's behavioral skills, such as impulse control, social adjustment and self-regulation, among others. General findings reveal the importance of early stimulation of executive functions, and its benefits in educational contexts. Furthermore, the before mentioned findings suggest the potential contribution of EFSP programs on everyday school practice. The ecological validity of this study allows an extrapolation of our results to school settings and constitutes an interesting alternative to 
traditional laboratory experiments for future studies. Finally, these results may constitute an interesting ground to reinforce the implementation of EFSPs in school contexts, because of the observed benefits on executive performance.

Keywords: executive functions; academic performance; early stimulation; cognitive developement; mixed factorial design.

\section{Introducción}

Durante la última década se ha observado un creciente interés en el estudio de las funciones ejecutivas (FE), principalmente en la infancia, por su asociación con competencias cognitivas y sociales (Clark, Pritchard y Woodward, 2010) y por su relación con el rendimiento académico (Diamond y Lee, 2011). La evidencia muestra que un buen desarrollo de las FE en la infancia permite predecir el éxito escolar (Canet, Andrés, García, Richard's y Burin, 2017; Portellano, Martínez y Zumárraga, 2009). Por ejemplo, se ha asociado la flexibilidad cognitiva $y$ el control inhibitorio con la predicción del desempeño matemático temprano (Clark et al., 2010; Fitzpatrick, McKinnon, Blair y Willoughby, 2014) y la inhibición cognitiva con el desarrollo de habilidades lectoras (Escobar et al., 2018), lo que puede proveer ventajas comparativas a los niños que han sido estimulados tempranamente (Bernal y Rodríguez, 2014; Blair y Diamond, 2008; Röthlisberger, Neuenschwander, Cimeli, Michel y Roebers, 2012). Cabe destacar que al estudiar los efectos de las FE sobre el desempeño académico es importante considerar el rol mediador del nivel socioeconómico (NSE), encontrándose que a mayor NSE mejor desempeño ejecutivo (Escobar et al., 2018; Ghiglione, Arán, Manucci y Apaz, 2011). De acuerdo con lo anterior, el desarrollo precoz de las FE puede favorecer el éxito escolar, período en el que las diferencias personales comienzan a incidir en las habilidades y trayectorias académicas (Best, 2012). Por lo que, estimular en el instante apropiado es fundamental para que tales capacidades funcionen de manera óptima.

Al establecer criterios para estimular las FE, resulta relevante comprender el constructo y situarlo en un modelo explicativo, dado que no existe consenso respecto a su definición (Wiebe et al., 2011). Esto puede deberse a que las FE dependen de múltiples procesos cognitivos, lo que dificulta determinar cuáles son sus componentes básicos y cómo se organizan en el cerebro (Van der Ven, Kroesbergen, Boom y Leseman, 2013).

Una forma de comprender las FE es considerar sus sustratos neuroanatómicos, los que se asocian principalmente a vías neuronales que involucran regiones corticales y subcorticales del lóbulo frontal. Estas conexiones neuronales se desarrollan paulatinamente desde la niñez a la adultez (Wiebe et al., 2011). La red neuronal involucrada en el funcionamiento de las FE incluye tres vías (Nigg y Casey, 2005): a) fronto estriada, b) fronto límbica y c) fronto cerebelosa. La vía fronto estriada está implicada en la habilidad de inhibir conductas y atender cuando se presentan distractores, favorecer el uso adecuado de la memoria de trabajo y permitir la estructuración de objetivos y planes. Ha sido asociada al denominado factor frío o cognitivo del circuito neural de las FE. Por otra parte, la vía fronto límbica subyace a los aspectos emocionales y motivacionales del comportamiento, considerando el control de impulsos o tendencias agresivas. Se asocia con el denominado factor caliente o emocional de la red de las FE. Por último, la vía fronto cerebelosa provee los circuitos neurales necesarios para el normal funcionamiento de las respuestas motoras y la distribución del tiempo, favoreciendo la organización de la conducta y el aprendizaje de rutinas y hábitos. Ha sido asociada con los aspectos psicomotores de la red neural de las FE.

Cabe resaltar la importancia de comprender la estructura de los circuitos neuronales de las FE a comienzos de la etapa escolar, con el fin de desarrollar propuestas eficaces de prevención, estimulación e intervención para niños 
con dificultades incipientes en esta dimensión cognitiva (Diamond, Barnett, Thomas y Munro, 2007).

\section{Las funciones ejecutivas: aspectos generales}

En términos generales, las FE incluyen múltiples funciones que ordenan y dirigen la totalidad de las operaciones cognoscitivas y conductuales. Integran la destreza para la iniciación de una tarea, inhibir comportamientos, elegir las acciones necesarias para alcanzar objetivos, elaborar estrategias apropiadas para resolver conflictos difíciles, diversificar la utilización de estrategias, analizar y juzgar el propio comportamiento y para ordenar y dirigir los procesos de memoria (Betancur, Molina y Cañizales, 2016; Isquith, Gioia y Espy, 2004; Richard's et al., 2017).

Como se señaló anteriormente, no existe consenso absoluto sobre la definición de las FE (Wiebe et al., 2011). No obstante, para los efectos del presente estudio se adopta la perspectiva genético-cultural de Alexander R. Luria y el paradigma histórico-cultural de Lev Vygotsky, quienes coinciden en describir el funcionamiento mental no solo desde una perspectiva biológica, sino también sociocultural (Vygotsky, 1978). Bajo este paradigma, las FE son concebidas como habilidades mentales de orden superior, autorreguladas, esenciales para el desarrollo y mediadas por el aprendizaje. Una de las principales bases de la perspectiva histórico-cultural es que el desarrollo mental de los niños no ocurre solo por instinto o maduración, sino más bien por la existencia de procesos objetivos de interacción comunicativa con su entorno (Luria, 1976). Por lo tanto, se hace fundamental entender la organización y funcionamiento cerebral desde sus etapas más tempranas.

Otros autores entienden las FE como una familia de procesos mentales top-down, fundamentales para la concentración, atención y situaciones donde las respuestas automáticas no resultan ser del todo eficaces (Diamond,
2013; Miller y Cohen 2001). Por su parte, como se señaló anteriormente, las FE pueden ser clasificadas en frías y calientes (Zelazo y Müller, 2002). Las FE frías están relacionadas con tareas que demandan poco control emocional, como la resolución de problemas, $\mathrm{y}$ las calientes, con tareas que requieren de reacciones y control emocional.

Pese a los múltiples conceptos que definen las FE, existe consenso en tres puntos centrales, observados en el modelo de Miyake et al. (2000), los que consideraron la unidad y separabilidad de estas $\mathrm{FE}$, incluyendo tres factores: a) memoria de trabajo, b) control inhibitorio y c) flexibilidad cognitiva. La primera se refiere a un componente implicado en la manipulación de la información entrante, su adaptación y manipulación mental. La segunda, a la capacidad para ignorar los estímulos irrelevantes y suprimir las respuestas prepotentes. Finalmente, la flexibilidad cognitiva implica procesos de atención en diferentes demandas de tareas, operaciones y juegos mentales (Miyake y Friedman, 2012). Las dimensiones descritas subyacen a tareas más complejas, tales como planificación, resolución de conflictos y razonamiento (Collins y Koechlin, 2012).

En el contexto escolar estas habilidades se hacen necesarias, dado que los estudiantes deben ser capaces de concentrarse, ignorar distracciones, atender a sus profesores, seguir las reglas de la clase, interactuar de manera positiva con sus compañeros, esperar recompensas e inhibir impulsos de jugar o agredir a otros. Por ello, no es sorprendente que las FE se consideren una herramienta clave para la preparación para la escuela y se relacionen con el desempeño escolar (Bernal y Rodríguez, 2014; Bernal, Rodríguez, González y Torres, 2018; Blair y Razza, 2007; Diamond, 2013; Diamond y Lee, 2011;).

\section{Estrategias de estimulación de las funciones ejecutivas}

Nigg y Casey (2005) describen la organización neuroanatómica de las FE sobre la base 
de tres circuitos cerebrales diferenciados (i.e., fronto-estriado, fronto-límbico y fronto-cerebeloso), los que pueden ser estimulados de manera específica considerando el rol diferencial que cumple cada uno de ellos (i.e., cognitivo, emocional, psicomotor).

\section{Estrategias de estimulación del componente cognitivo de las $\mathrm{FE}$}

El programa conducido por Espinet, Anderson y Zelazo (2013) para fomentar la reflexión en el procesamiento de la información a edades tempranas ha demostrado ser exitoso durante los primeros años escolares. Tal programa se basa principalmente en el uso de la memoria de trabajo para la formulación, selección y mantenimiento de ideas. En particular, el ejercicio que ellos proponen es ordenar tarjetas bivalentes, donde una primera dimensión corresponde a color y otra a forma, por ejemplo conejos rojos y barcos azules. Considerando esto, se establecen reglas jerárquicas que resuelven el conflicto de los estímulos bivalentes. De este modo, los menores deben reflexionar sobre el criterio de organización (i.e., forma o color), antes de iniciar la tarea. Tras la intervención, los autores observaron resultados positivos en la capacidad de reflexión de los estudiantes, especialmente en aquellos que recibieron feedback inmediato para corregir errores. Lo anteriormente expresado evidencia que la retroalimentación favorece la reflexión espontánea y pone de manifiesto por qué esta habilidad se considera esencial para el desarrollo temprano de las FE (Espinet et al., 2013).

El programa Stop, Think, Do (Detente, Piensa, Actúa; Petersen, 1995) es otra estrategia de estimulación de FE que ha demostrado ser útil para desarrollar habilidades de autocontrol, percepción y comunicación (i.e., Stop), habilidades cognitivas para resolver problemas (i.e., Think) y habilidades conductuales (i.e., Do). Este programa tiene como principal objetivo suprimir las emociones de todas las áreas problemáticas (e.g., conduc- tuales, de aprendizaje, sociales y de ánimo) y redirigir la energía hacia la resolución de problemas. En la etapa Stop, las emociones se identifican y se expresan tempranamente, asegurándose que el cerebro tenga la oportunidad de pensar acerca de las opciones para resolver el problema, con el fin posterior de llevarlas a la acción. El Programa Stop, Think, Do incorpora señales visuales, auditivas $\mathrm{y}$ táctiles para recordarle al niño que se detenga, además de estrategias de autocontrol para ayudarlos a pensar, y redirección conductual para llevar a cabo estas estrategias en el hacer (Petersen, 1995).

Otra estrategia innovadora para la estimulación del componente cognitivo es la de meditación/relajación, cuyo objetivo es fortalecer la percepción y las capacidades atencionales, favoreciendo la conciencia sensorial, del entorno y de los otros (Diamond y Lee, 2011). El modelo de educación Montessori es un ejemplo de este enfoque, el que incentiva la concentración profunda, la integración cuerpo-mente y las prácticas de rutinas de atención plena, tales como the silence, caminar en una recta o caminar con un libro en la cabeza (Lillard, 2011). El objetivo de estos ejercicios es fortalecer las capacidades atencionales, con fases de concentración focalizadas en una tarea que sea atractiva y que implique un desafío para los estudiantes, favoreciendo así su atención sostenida. Estas prácticas cobran sentido cuando los estudiantes toman conciencia de sus vivencias sensoriales (e.g., texturas, olores, sonidos, sabores, movimientos corporales, entre otros) intentando así poner en contacto la mente y el cuerpo (Lillard, 2011). Otro ejemplo en esta misma línea es el de Oros (2008), quien empleó diversos métodos de relajación infantil que en su mayoría aluden al componente cognitivo, tales como técnicas de distracción cognitiva mediante estrategias de modelado, reforzamiento positivo, ensayo conductual, análisis de costos-beneficios, búsqueda de alternativas, juego de planificación, entre otros. Estos ejercicios buscan disminuir la aparición de respuestas agresivas 
frente a situaciones de tensión, ejercitando la reflexión infantil, trabajando el control de impulsos y la toma de decisiones (Oros, 2008). Los resultados evidenciaron que, pese al corto tiempo de intervención (i.e., 6 sesiones de 45 minutos), se obtuvo un impacto positivo en los niños, incrementando la relajación y reduciendo en un $40 \%$ las conductas agresivas en el contexto escolar.

La práctica budista constituye otro ejemplo en que la meditación posibilita la estimulación de funciones cognitivas como la atención, en tanto la persona se centra en una sola idea, experimentando su respiración y con esto fortaleciendo su capacidad de atención visoespacial (Jha, Krompinger y Baime, 2007).

\section{Estrategias de estimulación del componente emocional de las FE}

El programa Herramientas de la Mente fundado en el enfoque de Vygotsky y Luria, que permite desarrollar conductas autorreguladas, es un buen ejemplo de cómo estimular el componente emocional de las FE (Bodrova, Leong y Akhutina, 2011). Este programa enfatiza el uso del juego como una acción primordial en los estudiantes, utilizando tareas didácticas y una valoración diaria activa. Una de las cualidades diferenciadoras de esta propuesta es la integración de componentes cognitivos y socioafectivos de las FE, mediante actividades simbólico-imaginarias que forman parte del currículum académico, con el objetivo de practicar la autorregulación y favorecer aprendizajes académicos, como las matemáticas, la lectoescritura y las habilidades sociales y emocionales (Bodrova et al., 2011).

Una de estas prácticas han sido los mediadores externos, que incluyen tarjetas con las palabras oído y boca, las que son utilizadas por los estudiantes para señalar su rol en el juego y un mediador que los ayuda en su proceso de autorregulación de dos formas complementarias. Por una parte, los niños les recuerdan a sus compañeros qué rol están ejerciendo y con esto los regulan, y por otra, utilizan los mediadores como un recordatorio de sus propios papeles. El programa, a su vez, parte de la premisa que los docentes cuentan con las habilidades necesarias para realizar evaluaciones dinámicas e individualizadas que consideren y respeten las formas de aprendizaje de sus estudiantes (Bodrova et al., 2011).

Otro programa de estimulación del componente emocional de las FE en el aula, muy similar al Stop, Think, Do de Petersen, es el PATHS (trad. Promoción Alternativa Pensando en la Estrategia; Riggs, Greenberg, Kusché y Pentz, 2006), que consiste en capacitar a los docentes para fortalecer competencias de autorregulación, reconocimiento emocional y resolución de conflictos interpersonales en los estudiantes. Su principio base es que los niños experimentan y responden a sus emociones previo a conseguir regularlas, muchas veces de manera impulsiva y sin control top-down, debido a lo cual proponen como estrategias educativas la expresión emocional y el ejercicio del control consciente. Lo dicho anteriormente puede ser llevado a la práctica, por ejemplo, a través de un modelo de semáforo en el que la luz roja indica Stop!- detente, instando a los estudiantes a realizar una larga y profunda respiración, e identificar el problema y sus emociones asociadas. Análogamente, la luz amarilla representa slow down...piensa, donde los estudiantes deben analizar el problema, identificar las soluciones posibles y elegir la mejor alternativa. Finalmente, la luz verde invita a los estudiantes a poner en práctica la solución previamente seleccionada y evaluar su funcionamiento, incentivando la expresión verbal y la identificación de sus emociones, con el objetivo de fortalecer más aún la regulación emocional (Bernal y Rodríguez, 2014).

\section{Estrategias de estimulación del componente psicomotor de las $\mathrm{FE}$}

Existe evidencia que sugiere que el ejercicio aeróbico mejora sustancialmente la 
activación de la corteza prefrontal y las FE (Chaddock, Pontifex, Hillman y Kramer, 2011). Asimismo, se ha reportado que el movimiento cumple un rol esencial en el desarrollo cognoscitivo (Best, 2012) y que tanto las tareas motoras tempranas como las de atención sostenida, son fundamentales para el posterior desarrollo de las FE. Un ejemplo de ello son los juegos aeróbicos de los niños, que implican la interacción de múltiples FE, entre ellas el pensamiento estratégico orientado a un objetivo, cuyo sustrato neuroanatómico se encuentra asociado a circuitos neurales del lóbulo frontal. A su vez, estas actividades aeróbicas generan no solo cambios fisiológicos generales en el cuerpo (e.g., aumento del flujo sanguíneo) sino también cambios específicos en el cerebro (Best, 2012).

El trabajo reciente de Martin et al. (2015) pone de manifiesto la relevancia de estimular el componente psicomotor de las FE, luego de observar los efectos positivos de un programa de actividad física en la memoria de trabajo y la flexibilidad cognitiva en un grupo de adolescentes. Previamente, Lakes y Hoyt (2004) reportaron mejoras en las capacidades de autorregulación, habilidades prosociales y autoestima, asociadas a la práctica de artes marciales versus actividad física tradicional. La evidencia descrita sugiere que la práctica de ejercicios que enfatizan habilidades de autocontrol y disciplina, tales como las artes marciales o el mindfulness, favorecen el desarrollo de las FE (Diamond y Lee, 2011; Jha et al., 2007; Lakes y Hoyt, 2004; Oros, 2008).

Con todo, es posible evidenciar que el contexto escolar es un espacio favorable para la implementación de programas de estimulación temprana con los estudiantes (Diamond, 2013; Diamond y Lee, 2011), requiriendo cambios mínimos y de bajo presupuesto para fortalecer los múltiples componentes de las FE, utilizando el juego (Bodrova et al., 2011), el razonamiento (Espinet et al., 2013), la relajación y meditación (Lillard, 2011), el uso de pensamiento estratégico (Riggs, et al., 2006) y las técnicas de entrenamiento psicomotor
(Lakes y Hoyt, 2004).

A la luz de estos antecedentes que confirman la importancia que tiene el desarrollo y estimulación temprana de las FE, la presente investigación tiene por objetivo estudiar el efecto de un programa de estimulación de las FE sobre el desempeño ejecutivo de estudiantes de primero básico y su eventual relación con el rendimiento académico. En particular, se espera que la implementación de un programa de estimulación de las FE genere un efecto significativo en la mejora del desempeño ejecutivo de los estudiantes. Del mismo modo, se espera que tal efecto sea observado además en la dimensión de rendimiento académico.

\section{Método}

\section{Participantes}

En este estudio participaron 43 estudiantes de primero básico de la Escuela Naciones Unidas de la Comuna de Valparaíso, Chile. El tipo de muestreo fue no probabilístico e intencionado. En cuanto a la dimensión intergrupo el primer año básico A fue considerado como condición control (i.e., sin intervención) y el paralelo B asumió la condición experimental (i.e., programa de estimulación de las FE). Además, al interior de cada grupo todos los sujetos fueron evaluados en dos ocasiones (i.e., pre y post), lo que constituye la dimensión intrasujeto. El grupo control estuvo conformado por 18 estudiantes (mujeres: $\mathrm{N}$ =9; edad: $\mathrm{M}=6.3$ años, $\mathrm{DS}=.5$; hombres: $\mathrm{N}=9$; edad: $\mathrm{M}=6.7$ años; $\mathrm{DS}=.7$ ), mientras que el grupo experimental se conformó por 25 estudiantes (mujeres: $\mathrm{N}=9$, edad: 6.1 años, $\mathrm{DS}=.3$; hombres: $\mathrm{N}=19$, edad: $\mathrm{M}=$ 6.6 años, DS = .9). Los criterios de inclusión contemplaron: a) ser estudiantes de primer año de enseñanza básica en un establecimiento educacional (EE) con un índice de vulnerabilidad escolar alto, b) tener entre 6 y 7 años de edad durante la aplicación del programa de intervención, c) no poseer diagnóstico previo 
o actual de algún trastorno del neurodesarrollo. Este estudio contó con la autorización oficial del EE y además con el consentimiento informado de los padres y los participantes, y se desarrolló según los lineamientos éticos de la Declaración de Helsinki para experimentación que involucra seres humanos.

\section{Diseño}

El presente estudio considera un diseño factorial mixto donde se estudia el efecto de un factor intergrupo (i.e., grupo control vs. grupo experimental), un factor intrasujeto (i.e., medidas pre y post intervención) y el efecto combinado de ambos factores, sobre el desempeño ejecutivo de estudiantes de primer año de educación básica. Desde la perspectiva del factor intergrupo, se podría considerar que el diseño es cuasi-experimental, dado que no existe la posibilidad de aleatorizar los participantes a los distintos grupos conformados (i.e., control vs. experimental). No obstante, el estudiar de manera simultánea el efecto de un factor intergrupo y de uno intrasujeto define al diseño como de tipo mixto. Más aún, un diseño mixto constituye la opción metodológica apropiada cuando se busca estudiar la existencia de efectos de interacción (i.e., combinados) entre factores intergrupos e intrasujetos (Balluerka y Vergara, 2002). Dada su naturaleza, los diseños mixtos pertenecen necesariamente a la categoría de diseños factoriales.

\section{Instrumentos y medidas}

\section{Subprueba de Funciones Ejecutivas del Test de Evaluación Neuropsicológica Infantil (TENI)}

Para evaluar las FE de los escolares de la muestra se empleó el Test de Evaluación Neuropsicológica Infantil TENI (Tenorio et al., 2012). Está compuesto por ocho subpruebas independientes entre sí, las cuales se presentan como juegos en una tablet, para evaluar cinco funciones cognitivas: a) atención, b) desarrollo viso-espacial, c) sistemas de memoria, d) lenguaje y d) funciones ejecutivas.

El TENI ha sido estandarizado en Chile, manifestando niveles de confiabilidad que fluctúan entre .80 y .90 ( $\alpha$ de Cronbach) Cronbach's $\alpha=.80$ y Cronbach's $\alpha=.90$ para las escalas que han sido contempladas en este estudio. Está orientado a la norma y ha sido diseñado con una concepción modular funcional de la cognición. Se puede aplicar a niños desde los 3 años hasta los 9 años 11 meses, y su administración dura aproximadamente 30 minutos.

Particularmente en este estudio se consideró el rendimiento de los estudiantes en la subprueba de FE del TENI, que consta de cuatro componentes: a) Memoria de Trabajo (i.e., prueba Torpo el Topo Torpe), b) Seriación (i.e., prueba La Granja), c) Inhibición conductual (i.e., prueba Bzz! - Inhibición) y d) Teoría de la Mente (i.e., prueba Trini y Agu). La sumatoria de las puntuaciones individuales obtenidas en estas pruebas da origen al puntaje total de la variable Funciones Ejecutivas del TENI de cada sujeto.

\section{Rendimiento académico}

Esta variable se obtuvo a partir de la media aritmética entre las calificaciones finales semestrales de los cursos de Lenguaje y Matemática de cada estudiante. Se obtuvieron indicadores correspondientes al primer y segundo semestre.

\section{Procedimiento}

Inicialmente se efectuó una reunión con el equipo directivo de la escuela a intervenir, explicando los objetivos de la investigación, el procedimiento a efectuar, los participantes requeridos, la duración y los resultados esperados. Una vez aprobada la propuesta, se participó de una reunión de apoderados orientada a explicar el sentido del estudio y obtener su consentimiento informado. 
Todos los participantes del estudio fueron evaluados en dos ocasiones, la primera antes de llevar a cabo la intervención en el grupo experimental y la segunda, una vez concluida la intervención. Luego de la primera evaluación, el grupo experimental (i.e., primero básico B) participó del programa de estimulación de las FE, mientras que el grupo control (i.e., primero básico A) realizó sus clases de manera habitual. El programa fue conducido por un educador diferencial, con apoyo de la profesora y la asistente de aula. La duración de la intervención fue de 12 semanas, dos veces por semana y durante dos horas pedagógicas en aula. En estas sesiones se planificaron acciones incorporando al currículum académico actividades articuladas que estimularan las FE en sus tres componentes (i.e., cognitivo, afectivo y conductual), mediante estrategias de modelamiento, feedback y refuerzo positivo, meditación, técnicas de respiración, análisis de las experiencias, preguntas para favorecer la metacognición y juegos. Cada sesión se planificó considerando los fundamentos de la clase, a saber: a) Inicio, presentando los objetivos de la sesión, b) Desarrollo, llevando a cabo las actividades planificadas y haciendo explícitas las estrategias utilizadas para la estimulación de las FE en cada actividad y c) Cierre, donde se retomaba el objetivo, intencionándose un proceso metacognitivo orientado a que los estudiantes reflexionaran sobre su propio funcionamiento y fueran capaces de determinar sus niveles de logro asociado a las actividades y las FE estimuladas.

Para la estimulación de las FE, se hizo una adaptación del modelo Stop, Think, Do (Petersen, 1995) y del programa PATH (Riggs et al., 2006). La adaptación consideró cinco pasos: a) Parar, b) Escuchar, c) Pensar (o hacer un Plan de Acción), d) Hacer y e) Revisar. Esta dinámica se usó de forma transversal en todo el proceso de intervención con el objetivo de favorecer procesos reflexivos en el accionar y procesos metacognitivos de control de las actividades realizadas. Por lo tanto, se fue mecanizando y estableciendo dentro de la rutina de la sala de clases, generando que cada vez que se iniciaba una actividad se realizaban los cinco pasos, creando un modelamiento en el aula.

El componente cognitivo de las FE se trabajó desde dos aspectos. El primero relacionado con la propia regulación del comportamiento, potenciando las capacidades de regulación y monitoreo comportamental en los participantes del grupo experimental. El segundo aspecto, vinculado con actividades de inhibición y auto-monitoreo, en que los participantes del grupo experimental debían buscar de forma personal una regulación cognitiva para iniciar, mantener y finalizar, junto con monitorear su funcionamiento en cada actividad propuesta. Por ejemplo, una actividad consistió en que el educador contaba una historia $y$, en la medida que esta transcurría, se le pedía a los estudiantes que fueran inventando e integrando partes, de modo de mantenerlos atentos, esperando su turno para hablar e ir siguiendo la historia, favoreciendo así el funcionamiento de la memoria de trabajo. En paralelo, y como segundo eje, se trabajó desde la metacognición, vinculada a la habilidad de cada niño para controlar, gestionar los procesos cognitivos y resolver problemas de manera efectiva. En este eje se buscó estimular las FE de iniciativa, memoria de trabajo, planificación y monitoreo de la tarea. Cada actividad tenía un inicio (i.e., luz roja), donde se detenían los trabajos que se estaban realizando y se daban las instrucciones de la nueva tarea y los lineamientos escritos y orales, así como también se les pedía a los estudiantes del curso que verbalizaran lo que habían entendido y debían realizar. Posteriormente, se procedía a un tiempo para planificar el accionar (i.e., luz amarilla), en forma personal o grupal según la tarea, para finalmente pasar al hacer y revisar (i.e., luz verde). Cada momento fue acompañado de carteles que orientaban la ejecución de la actividad.

El componente emocional de las FE se estimuló basándose principalmente en el 
Programa PATH (Riggs et al., 2006), en torno al uso de estrategias de autocontrol, autorregulación y de actividades de reconocimiento de emociones y de solución de problemas interpersonales. Además, se buscó fortalecer en los estudiantes las capacidades de organización y planificación de diferentes acciones para resolver tareas y conflictos, eligiendo la mejor alternativa, pero también recurriendo a otras alternativas si no se tenía éxito en un primer momento. Esto se trabajó a través de la elaboración de un títere, en la cual mediante la creación artística los estudiantes podían generar historias, resolver conflictos, visitar el pasado y anticipar el futuro, conectar su historia con la de su compañero e incorporar emociones.

Finalmente, el componente psicomotor de las FE, fue estimulado mediante actividades motoras y/o de relajación/meditación, dependiendo del nivel de activación de los niños en distintos momentos de la clase, generalmente al inicio de la jornada, antes de comenzar los quehaceres escolares, se realizaban actividades para activar el cuerpo y la mente, tales como saltar abriendo y cerrando las extremidades, correr en el lugar y mover cosas con peso, entre otras. Luego del recreo, para controlar los impulsos y disminuir los niveles de activación con que los niños llegaban del patio, se realizaban actividades de mindfulness, tales como postura de meditación, enseñándoles a fijar su atención en la respiración, de escaneo corporal, promoviendo la conciencia sensorial y el juego del silencio para iniciarse en técnicas de concentración.

El programa de estimulación de las FE aquí presentado, contempló además, un trabajo quincenal con la profesora y la asistente de aula del grupo experimental, cuyos objetivos eran, por una parte, entregarles herramientas para que ellas siguieran con la estimulación a lo largo de la semana, y por otra, retroalimentar las actividades planificadas por ellas para el trabajo diario con los estudiantes en aula. Todo esto con el objetivo de integrar en el quehacer diario de las docentes y los estu- diantes el comportamiento regulado y planificado.

\section{Análisis de datos}

Se reportan estadísticos descriptivos (i.e., $M, D S)$ de las variables dependientes estudiadas, para las medidas pre y post en los grupos control y experimental, respectivamente. La medida pre permitió establecer una línea de base para determinar los niveles de desempeño en FE de ambos grupos, mientras que la medida post permitió comparar el efecto de la presencia o ausencia del programa de estimulación sobre la línea de base establecida. Lo mismo ocurre en relación con las medidas pre y post del rendimiento académico, que corresponden al promedio de las calificaciones finales entre lenguaje y matemática del primer y segundo semestre, respectivamente.

Para contrastar los efectos principales de los factores intergrupos, intrasujetos y de interacción, se efectuó un análisis de la varianza mixto (ANOVA mixto). Para todas las pruebas de hipótesis efectuadas se consideró un nivel de significancia $\alpha=.05$. Previo a la realización de los análisis estadísticos inferenciales se efectuó el respectivo contraste de los supuestos a la base de las pruebas paramétricas. En particular, normalidad univariada (Shapiro-Wilk) y homogeneidad de las varianzas (Levene test). La prueba de esfericidad (W de Mauchly) no es requerida, en tanto el factor intrasujeto tiene solamente dos mediciones (pre y post) y en ese caso especial, el supuesto de esfericidad se cumple siempre.

Se reportan los estadísticos de prueba, sus valores $p$ respectivos y los tamaños de efecto observados (Cohen, 1988). Todos los análisis estadísticos fueron realizados con el software JASP Versión 0.7.5.5 (JASP Team, 2016).

\section{Resultados}

Las medias $(M)$ y desviaciones estándar $(D S)$ para las FE y rendimiento académico (RA) son expuestas en las Tablas 1 y 2 , respec- 
tivamente. Estos estadísticos descriptivos se exponen para ambos factores de manera simultánea, es decir, intergrupo (i.e., Control vs. Experimental) e intrasujeto (i.e., Pre vs. Post).

Tabla 1

Estadisticos descriptivos de las Funciones Ejecutivas (FE) de las mediciones pre y post para los grupos Control y Experimental.

\begin{tabular}{lc|cccc}
\multicolumn{1}{c}{} & \multicolumn{1}{c}{ Factor Intergrupos } & M & SD & N \\
\cline { 3 - 6 } Factor & Pre & Control & 8.64 & 2.56 & 18 \\
Intrasujetos & & Experimental & 7.97 & 3.39 & 25 \\
\cline { 3 - 5 } & Post & Control & 8.64 & 2.19 & 18 \\
\cline { 3 - 5 } & & Experimental & 10.83 & 2.38 & 25
\end{tabular}

Tabla 2

Estadísticos descriptivos del Rendimiento Académico (RA) pre y post para los grupos Control y Experimental.

\begin{tabular}{cccccc}
\multicolumn{1}{c}{} & Factor Intergrupos & $\mathrm{M}$ & $\mathrm{SD}$ & $\mathrm{N}$ \\
\cline { 3 - 6 } $\begin{array}{c}\text { Factor } \\
\text { Intrasujetos }\end{array}$ & & Control & 5.58 & 1.00 & 18 \\
& Post & Experimental & 5.18 & .84 & 25 \\
\cline { 3 - 6 } & & Control & 5.59 & .99 & 18 \\
& & Experimental & 5.15 & 1.11 & 25
\end{tabular}

En los análisis estadísticos realizados no se transgredió ninguno de los supuestos a la base de las pruebas paramétricas, pudiendo asumirse la existencia de normalidad univariada (Shapiro-Wilk test) y homogeneidad de las varianzas (Levene test).

Respecto de las FE se observa un efecto de interacción entre los factores intergrupo e intrasujeto, que resulta ser estadísticamente significativo $\left[\mathrm{F}(1,41)=7.399, p=.010, \eta^{2}\right.$ $=0.153]$. Lo anterior permite señalar que el desempeño de los estudiantes en la medida de las FE resulta ser significativamente mejor en la medición post para el caso del grupo experimental $[\mathrm{F}(1)=17.675, p<.001)$. Cabe señalar que la magnitud de las diferencias pre y post en el desempeño de los estudiantes del grupo experimental en las FE refleja un tamaño del efecto que se considera grande. $\mathrm{Al}$ respecto, los criterios de Cohen (1988) establecen un $\eta_{p}^{2}=.14$ como valor de referencia para determinar que el efecto de una intervención pueda ser considerado grande. En cuanto al grupo control, no se observan diferencias entre las medidas pre y post. Este hallazgo permite sugerir que la estimulación de las FE ejerció un efecto importante en la mejora de estas funciones en los estudiantes que participaron del programa anteriormente descrito (Ver Tabla 3 y Figura 1). 
Tabla 3

Resultados ANOVA mixto con Interacción Factores Inter e Intrasujetos para las Funciones Ejecutivas (FE).

\begin{tabular}{c|cccccc} 
& $\begin{array}{c}\text { Suma de } \\
\text { cuadrados }\end{array}$ & gl & $\begin{array}{c}\text { Media } \\
\text { cuadrática }\end{array}$ & $\mathbf{F}$ & $\mathbf{p}$ & $\mathbf{\eta}^{\mathbf{2}} \mathbf{p}$ \\
\hline Factor Intrasujetos & 42.740 & 1 & 42.740 & 7.399 & $.010^{* *}$ & .153 \\
$\begin{array}{c}\text { Factor Interacción } \\
\text { Intrasujetos X Intergrupos }\end{array}$ & 42.740 & 1 & 42.740 & 7.399 & $.010^{* *}$ & .153 \\
& & & & & &
\end{tabular}

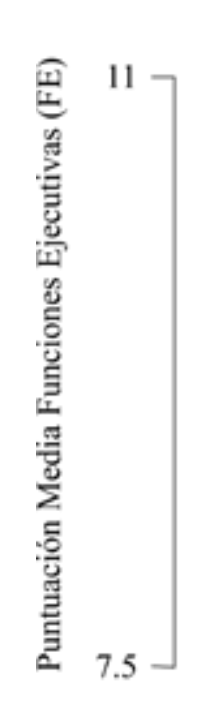

Factor Intergrupos

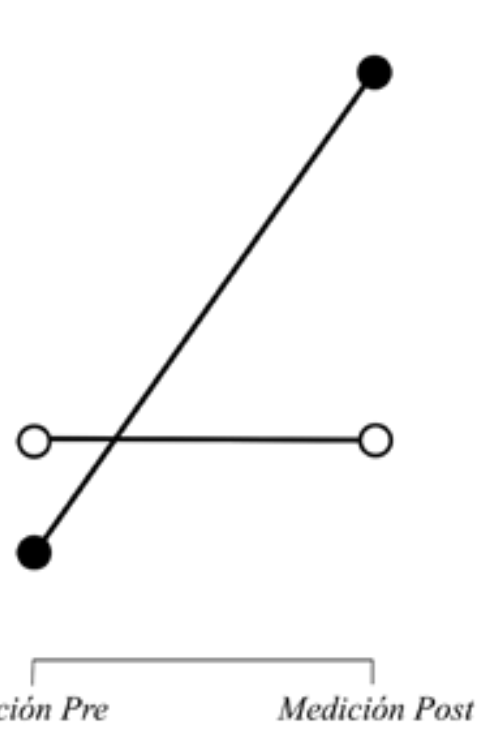

Control

\section{Factor Intrasujetos}

Figura 1. Interacción Factor Intra e Intersujetos para las Funciones Ejecutivas (FE).

Un hallazgo interesante, que se considera relevante destacar, es la existencia de un efecto de interacción para el componente de las FE de Control Inhibitorio (CI) que resulta ser estadísticamente significativo $[\mathrm{F}(1,41)=4.489, p$ $\left.=.033, \eta_{p}^{2}=.106\right]$. El tamaño del efecto de la interacción puede ser considerado cercano a grande, de acuerdo con los criterios de Cohen (1988). Es decir, se observó que el desempeño de los estudiantes en la medida de CI resulta ser significativamente mejor en la medición post para el caso del grupo experimental $[\mathrm{F}(1)$ $=13.221, p<.01)$. Este hallazgo será incorporado y discutido en la sección Discusión.
En contraposición a lo observado para el caso de las FE, el RA no refleja un efecto de interacción entre los factores intergrupo e intrasujeto $\left[\mathrm{F}(1,41)=.048, p=.828 n s ., \eta_{\mathrm{p}}^{2}\right.$ $=.001]$. Lo anterior permite señalar que no se observan diferencias estadísticamente significativas en el promedio de las calificaciones finales de Lenguaje y Matemática del primer versus el segundo semestre (Pre vs. Post), tanto en el grupo control $[\mathrm{F}(1,41)=.011, p=$ .920 ns.] como en el experimental $[\mathrm{F}(1,41)=$ $.046, p=.831 \mathrm{~ns}$.$] .$

Los resultados generales sugieren que la participación en el programa de estimulación 
de las FE ejerce un efecto importante en la mejora de este tipo de funciones, pero no así en el RA. En la sección Discusión se profundizará en los alcances e importancia de estos resultados.

\section{Discusión}

Esta investigación evaluó el efecto de un programa de estimulación de los tres circuitos de las FE en estudiantes chilenos de primero básico, sobre el propio desempeño ejecutivo y sobre el RA. Los hallazgos generales sugieren que la estimulación de las FE ejerce un impacto positivo en el desempeño ejecutivo. Lo anterior se sustenta en los efectos de interacción observados, donde el grupo experimental manifestó mejoras importantes post intervención, hecho que no se observó en el grupo control. Tales resultados satisfacen uno de los objetivos de esta investigación y ponen de manifiesto la relevancia de la estimulación de las FE en la infancia temprana y los beneficios que esto puede reportar (Bernal y Rodríguez, 2014; Röthlisberger, et al., 2012; Blair y Diamond, 2008). Un detalle metodológico importante es que la intervención se realizó en el aula, en un contexto en que los estudiantes tienen sus lecciones cotidianas, lo que le otorga al presente estudio una excelente validez externa. Ello sirve de argumento para sugerir que este tipo de intervenciones puedan ser incorporadas por los profesores en sus prácticas docentes habituales y de ese modo, contribuir a la estimulación temprana de las FE en sus estudiantes (Puebla y Talma, 2011).

Los presentes hallazgos están en línea con aquellos reportados por Arán y Richaud (2011) y los de Ghiglione et al. (2011), quienes reportan la eficacia de la estimulación de las FE en el desarrollo del desempeño ejecutivo y funciones cognitivas generales. Una implicancia interesante de los estudios recién mencionados es que la estimulación de las FE resulta apropiada para trabajar con niños en condición de pobreza y es pertinente al trabajo educativo por la facilidad para adecuarla a los contenidos curriculares. Lo anterior permite sostener que un programa de estimulación de las FE como el presentado en este estudio refleja un impacto positivo en poblaciones de niños en riesgo de pobreza. Este hecho cobra mayor relevancia si consideramos que es conocida la existencia de una asociación inversa entre la condición de pobreza y las destrezas cognitivas (Ghiglione et al., 2011).

En cuanto al efecto del programa sobre el RA, los resultados observados no arrojan evidencia que ponga de manifiesto el beneficio de dicho programa en el RA (i.e., medido como el promedio entre las calificaciones finales semestrales de los cursos de Lenguaje y Matemática de cada estudiante). Si bien este hallazgo resulta contradictorio con literatura reciente que reporta la existencia de un efecto de la estimulación temprana de las FE sobre el rendimiento académico (Clark et al., 2010; Escobar et al., 2018; Fitzpatrick et al., 2014; Risso et al., 2015), una posible explicación a este fenómeno es que la medida empleada como indicador del RA no refleje de buena manera las características de las FE. Además, el análisis por separado del indicador utilizado (i.e., calificación final semestral en matemática y calificación final semestral en lenguaje) tampoco arroja el efecto hipotetizado. En este sentido, una explicación que cobra mayor relevancia dice relación con el Índice de Vulnerabilidad Escolar (IVE) del EE en el que se efectuó la intervención, el cual alcanza un IVE de $90.5 \%$, lo que se asocia a elevados niveles de pobreza de sus estudiantes (Cornejo et al., 2005). Autores como Escobar et al. (2018) sostienen que la asociación entre FE y pobreza es compleja, afirmando que en contextos de mayor vulnerabilidad se observan bajos rendimientos en pruebas asociadas al desempeño ejecutivo en comparación con grupos de menor riesgo. Musso (2010), por su parte, señala que el alto índice de vulnerabilidad está asociado a una disminución del desarrollo neurocognitivo en niños. Lo anterior cobra especial relevancia si consideramos que las calificaciones semes- 
trales de los estudiantes (i.e., indicadores de RA) podrían estar influenciadas en mayor medida por el contexto de precariedad permanente en que se desarrolla su proceso formativo y no reflejen necesariamente el efecto del programa implementado, cuya duración fue de solo 12 semanas. Un hecho que apoya esta tesis es lo reportado por Jadue (2002), quien observó que las calificaciones en primero básico muchas veces están influidas por múltiples variables que no necesariamente responden a aspectos propios del estudiante, realidad que se acentúa en contextos de vulnerabilidad social. Es un hecho demostrado que el NSE restringe las oportunidades educativas a las que pueden acceder los niños debido a la alta segregación y selección de los colegios (Escobar et al., 2018; Hernández y Raczynski, 2015; Valenzuela, Bellei y Ríos, 2014). Una manera de soslayar esta situación sería implementando el programa aquí presentado en una institución educacional que no presente tales características de vulnerabilidad. Esta debilidad asociada al presente estudio puede constituir, sin embargo, un objetivo a estudiar en investigaciones futuras.

Respecto al análisis por componentes del funcionamiento ejecutivo (i.e., memoria de trabajo, seriación, inhibición conductual y teoría de la mente), se observa un efecto diferencial del programa implementado sobre el control inhibitorio de los estudiantes. Es conocido que, ontogenéticamente, la región prefrontal del cerebro es una de las últimas en alcanzar su madurez, entre la segunda y tercera década de vida (Fuster, 2015). En particular, la región orbitofrontal es la que se encuentra vinculada a aspectos del comportamiento, tales como inhibición conductual y control de la impulsividad, entre otros, y como ya se ha mencionado, los contextos de pobreza se asocian a una disminución del desarrollo neurocognitivo en niños. A la luz de estos antecedentes, los resultados observados en este estudio ponen de manifiesto el efecto positivo que posee la estimulación temprana de las FE sobre el control inhibitorio, lo que impacta positivamente en los aspectos comportamentales asociados al manejo de impulsos, comprensión de normas, adecuación y adaptación social. Tales aspectos resultan esenciales para una integración adecuada del niño al contexto educativo, especialmente en situaciones de vulnerabilidad socioeconómica, lo que podría constituir un aporte especial del presente trabajo.

En otro ámbito, la mayoría de las investigaciones sobre estimulación de las FE, han efectuado intervenciones focalizadas en un circuito único de estas, centrándose ya en lo cognitivo (Diamond y Lee, 2011; Espinet et al., 2013; Lillard, 2011), ya en lo emocional (Bodrova et al., 2011; Riggs et al., 2006), ya en lo motor (Best, 2012; Lakes y Hoyt, 2004; Martin et al., 2015). En este sentido, el presente estudio efectúa una intervención integral en los tres circuitos de manera conjunta, lo que otorga mayores posibilidades de generalización a los hallazgos reportados. Del mismo modo, desde un punto de vista práctico, los hallazgos relevan la necesidad de continuar generando intervenciones para la estimulación de las FE en estudiantes, especialmente en los inicios de su periodo escolar (Bernal y Rodríguez, 2014). Por otra parte, se considera beneficioso aumentar los períodos de intervención y la frecuencia de éstos, con el objetivo de obtener un impacto no solo en los estudiantes, sino también dotar a los docentes de herramientas necesarias para organizar sus clases en función de la estimulación de las FE, y con ello, estimular sistemáticamente a los estudiantes desde la rutina escolar (Raver et al., 2011).

Un área relevante de explorar en estudios venideros sería recoger información respecto de las prácticas parentales de los padres y apoderados de los estudiantes, con el fin de conocer qué prácticas son las que en mayor medida contribuyen al desarrollo de las FE en otros espacios, como el hogar (Bernal et al., 2018). Además, otras posibles variables a considerar son el nivel socioeconómico, el bilingüismo y los contextos culturales (Flores, Castillo y Jiménez, 2014). 


\section{Conclusiones}

Este estudio resulta relevante como punto de partida para el diseño de programas de estimulación de las FE en el ámbito escolar, pues aporta evidencia que resalta los beneficios de la estimulación temprana de las FE para los estudiantes y su proceso de aprendizaje escolar (Bernal y Rodríguez, 2014; Blair y Diamond, 2008; Röthlisberger, et al., 2012). Esta propuesta resulta ser además válidamente ecológica e innovadora, pues sale del contexto de los estudios tradicionales de laboratorio. Finalmente, al ser una propuesta que estimula los tres circuitos neurales asociados a las FE (Bernal y Rodríguez, 2014) constituye un buen inicio para la incorporación de elementos neuropsicológicos en contextos educativos (Escobar et al., 2018).

\section{Referencias bibliográficas}

Arán, V. y Richaud, M.C. (2011). Efectos de un programa intervención para aumentar la reflexividad y la planificación en un ámbito escolar de alto riesgo por pobreza. Universitas Psychologica, 10(2), 341-354. http://dx.doi. org/10.11144/Javeriana.upsy10-2.epia

Balluerka, N. y Vergara, A.I (2002). Diseños de investigación experimental en psicología: modelos y análisis de datos mediante el SPSS 10.0. Madrid: Pearson Educación.

Bernal, F. y Rodríguez, M. (2014). Estimulación temprana de las funciones ejecutivas en escolares, una revisión actualizada. Revista de Orientación Educacional, 28(53), 15-24.

Bernal, F., Rodríguez, M., González, J. y Torres, A. (2018). Competencias parentales que favorecen el desarrollo de funciones ejecutivas en escolares. Revista Latinoamericana de Ciencias Sociales, Niñez y Juventud, 16(1), 163-176. http://dx.doi.org/10.11600/1692715x.16109

Best, J. (2012). Exergaming immediately enhances children's executive function. Developmental Psychology, 48(5), 1501-1510. http://dx.doi. org/org/10.1037/a0026648

Betancur, M., Molina, D. y Cañizales, L. (2016).
Entrenamiento cognitivo de las funciones ejecutivas en la edad. Revista Latinoamericana de Ciencias Sociales, Niñez y Juventud, 14(1), 359-368. http://dx.doi.org/10.11600/169 2715x.14124160615.

Bodrova, E., Leong, D.J. y Akhutina, T.V. (2011). When everything new is well-forgotten old: Vygotsky/Luria insights in the development of executive functions. En R.M. Lerner, J.V. Lerner, E.P. Bowers, S. Lewin-Bizan, S. Gestsdottir y J.B. Urban (Eds.), Thriving in childhood and adolescence: The role of self-regulation processes. New Directions for Child and Adolescent Development, 133, 11-28. http:// dx.doi.org/10.1002/cd.301

Blair, C. y Diamond, A. (2008). Biological processes in prevention and intervention: the promotion of self-regulation as a means of preventing school failure. Development and Psychopathology, 20(3), 899-911. http:// dx.doi.org/10.1017/S0954579408000436.

Blair, C. y Razza, R. (2007). Relating effortful control, executive function, and false belief understanding to emerging math and literacy ability in kindergarten. Child Development, 78(2), 647-663. http://dx.doi.org/10.1111/ j.1467-8624.2007.01019.x

Canet, L., Andrés, M.L., García, A., Richard`s, M.M. y Burin, D. (2017). Desempeño en memoria de trabajo e indicadores comportamentales: Relaciones entre medidas directas e indirectas. Interdisciplinaria, 34(2), 369-387. doi

Cohen, J. (1988). Statistical power analysis for the behavioral sciences. Hillsdale, N.J: L. Erlbaum Associates.

Collins, A. y Koechlin, E. (2012). Reasoning, Learning, and Creativity: Frontal Lobe Function and Human Decision-Making. PLoS Biology, 10(3), e1001293. http://dx.doi.org/10.1371/ journal.pbio. 1001293

Cornejo, A., Céspedes, P., Escobar, D., Núñez, R., Reyes, G. y Rojas, K. (2005). SINAE Sistema Nacional de Asignación con Equidad para Becas JUNAEB. Una nueva visión en la construcción de igualdad de oportunidades en la infancia. Junta Nacional de Auxilio Escolar 
y Becas. Dirección Nacional del Gobierno de Chile. Santiago, Chile.

Chaddock, L., Pontifex, M.B., Hillman, C.H. y Kramer, A.F. (2011). A review of the relation of aerobic fitness and physical activity to brain structure and function in children. Journal of the International Neuropsychological Society, 17(6) 975-985. http://dx.doi.org/10.1017/ S1355617711000567

Clark, C., Pritchard, E. y Woodward, J. (2010). Preschool executive functioning abilities predict early mathematics achievement. Developmental Psychology, 46(5), 1176. http:// dx.doi.org/10.1037/a0019672

Diamond, A. (2013). Executive Functions. Annual Review of Psychology, 64,135-168.http://dx.doi. org/10.1146/annurev-psych-113011-143750

Diamond, A., Barnett, W.S., Thomas, J. y Munro, S. (2007). Preschool Program Improves Cognitive Control. Science, 318(5855), 1387-1388. http://dx.doi.org/ 10.1126/science.1151148

Diamond, A. y Lee, K. (2011). Interventions shown to aid executive function development in children 4 to 12 years old. Science, 333(6045), 959-964. http://dx.doi.org/ 10.1126/ science. 1204529

Escobar, J.P., Rosas, R., Ceric, F., Aparicio, A., Arango, P., ... y Urzúa, D. (2018). El rol de las funciones ejecutivas en la relación entre el nivel socioeconómico y el desarrollo de habilidades lectoras y matemáticas. Cultura y Educación, 30(2), 368-392. http://dx.doi.org/1 0.1080/11356405.2018.1462903

Espinet, D., Anderson, E. y Zelazo, D. (2013). Reflection training improves executive function in preschool-age children: Behavioral and neural effects. Developmental Cognitive Neuroscience, 4, 3-15. http://dx.doi. org/10.1016/j.dcn.2012.11.009

Fitzpatrick, C., McKinnon, R.D., Blair, C.B. y Willoughby, M.T. (2014). Do preschool executive function skills explain the school readiness gap between advantaged and disadvantaged children?. Learning and Instruction, 30, 25-31. http://dx.doi.org/10.1016/j.learninstruc. 2013.11.003

Flores, J.C., Castillo, R.E. y Jiménez, N.A.
(2014). Desarrollo de funciones ejecutivas, de la niñez a la juventud. Anales de psicología, 30(2), 463-473. http://dx.doi.org/10.6018/ analesps.30.2.155471

Ghiglione, M., Arán, V., Manucci, V. y Apaz, A. (2011). Programa de intervención, para fortalecer funciones cognitivas y lingüísticas, adaptado al currículo escolar en niños en riesgo por pobreza. Interdisciplinaria, 28(1), 17-36.

Fuster, J. (2015). The prefrontal cortex.Amsterdam: Elsevier. http://dx.doi.org/10.1016/B978-0-12407815-4.00002-7

Hernández, M. y Raczynski, D. (2015). Elección de escuela en Chile: De las dinámicas de distinción y exclusión a la segregación socioeconómica del sistema escolar. Estudios Pedagógicos, 41, 127-141. doi:10.4067/S071807052015000200008

Isquith, P., Gioia, G. y Espy, K. (2004). Executive function in preschool children: Examination through everyday behavior. Developmental Neuropsychology, 26(1), 403-422. doi: $10.1207 / \mathrm{s} 15326942 \mathrm{dn} 26013$

Jadue, G. (2002). Factores psicológicos que predisponen al bajo rendimiento, al fracaso y a la deserción escolar. Estudios Pedagógicos, 28, 193-204. http://dx.doi. org/10.4067/S0718-07052002000100012

JASP Team. (2016). JASP (Versión 0.7. 5.5) [Computer software].

Jha, A. P., Krompinger, J. y Baime, M. J. (2007). Mindfulness training modifies subsystems of attention. Cognitive, Affective, \& Behavioral Neuroscience, 7(2), 109-119. http://dx.doi. org/10.3758/CABN.7.2.109

Lakes, D. y Hoyt, T. (2004). Promoting self-regulation through school-based martial arts training. Journal of Applied Developmental Psychology, 25(3), 283-302. http://dx.doi. org/10.1016/j.appdev.2004.04.002

Lillard, A. (2011). Mindfulness Practices in Education: Montessori's Approach. Mindfulness, 2(2), 78-85. http://dx.doi.org/10.1007/ s12671-011-0045-6

Luria, R. (1976). The Working Brain: An Introduction to Neuropsychology. New York: Basic Books. 
Martin, I., Chirosa, L., Reigal, R., Hernández, A., Juarez, R. y Guisado, R. (2015). Efectos de la actividad física sobre las funciones ejecutivas en una muestra de adolescentes. Anales de Psicología, 31(3), 962-971. doi:10.6018/ analesps.32.1.171601

Miller, E. y Cohen, J. (2001). An integrative theory of prefrontal cortex function. Annual Review of Neuroscience, 24(1), 167-202. http://dx.doi. org/10.1146/annurev.neuro.24.1.167

Miyake, A., Friedman, N., Emerson, M., Witzki, A., Howerter, A. y Wager, T. (2000). The Unity and diversity of executive functions and their contributions to complex "frontal lobe" tasks: A latent variable analysis. Cognitive Psychology, 41(1), 49-100. http://dx.doi.org/10.1006/ cogp.1999.0734

Miyake, A. y Friedman, N. (2012). The nature and organization of individual differences in executive functions: Four general conclusions. Current Directions in Psychological Science, 21(1), 8-14. http://dx.doi. org/ 10.1177/0963721411429458

Musso, M. (2010). Funciones ejecutivas: un estudio de los efectos de la pobreza sobre el desempeño ejecutivo. Interdisciplinaria, 27(1), 95-110.

Nigg, T. y Casey, J. (2005). An integrative theory of attention-deficit/ hyperactivity disorder based on the cognitive and affective neurosciences. Development and Psychopathology, 17(03), 785-806. http://dx.doi.org/10.1017/ S0954579405050376

Oros, L.B. (2008). Promoviendo la serenidad infantil en el contexto escolar: Experiencias preliminares en una zona de riesgo ambiental. Interdisciplinaria, 25(2), 181-195.

Petersen, L. (1995). Stop Think Do. En H. van Bilsen, P. Kendall y J. Slavenburg (Eds.) Behavioral Approaches for Children and Adolescents. Boston, MA: Springer. doi. org/10.1007/978-1-4757-9406-9_9

Portellano, J.A., Martínez, A. y Zumárraga, A. (2009). Evaluación de las funciones ejecutivas en niños. Madrid: TEA Ediciones.

Puebla, R. y Talma, M. (2011). Educación y neurociencias. La conexión que hace falta. Estudios
Pedagógicos, 37(2), 379-388. http://dx.doi. org/10.4067/S0718-07052011000200023

Raver, C., Jones, S., Li-Grining, C., Zhai, F., Bub, K. y Pressler, E. (2011). CSRP's Impact on Low-Income Preschoolers' Preacademic Skills: Self-Regulation as a Mediating Mechanism. Child Development, 82(1), 362-378. http:// dx.doi.org/10.1111/j.1467-8624.2010.01561.x Richard's, M.M., Vernucci, S., Zamora, E., Canet, L., Introzzi, I. y Guardia, J. (2017). Contribuciones empíricas para la validez de grupos contrastados de la batería de tareas de autorregulación Cognitiva (TAC). Interdisciplinaria, 34(1), 173-192. http://dx.doi.org/10.16888/ interd.2017.34.1.11

Riggs, R., Greenberg, T., Kusché, A. y Pentz, A. (2006). The mediational role of neurocognition in the behavioral outcomes of a social-emotional prevention program in elementary school students: Effects of the PATHS curriculum. Prevention Science, 7(1), 91-102. http://dx.doi.org/10.1007/s11121-005-0022-1

Risso, A., García, M., Durán, M., Brenlla, J., Peralbo, M. y Barca, A. (2015). Un análisis de las relaciones entre funciones ejecutivas, lenguaje y habilidades matemáticas. Revista de Estudios e Investigación en Psicología y Educación, 0(9), 73-78. http://dx.doi. org/10.17979/reipe.2015.0.09.577

Röthlisberger, M., Neuenschwander, R., Cimeli, P., Michel, E. y Roebers, C. (2012). Improving executive functions in 5-and 6-year-olds: Evaluation of a small group intervention in prekindergarten and kindergarten children. Infant and Child Development, 21(4), 411-429. http://dx.doi.org/10.1002/icd.752

Tenorio, M., Arango, P., Aparicio, A., Benavente, C., Thibaut., C. y Rosas, R. (2012). Test de Evaluación Neuropsicológica Infantil TENI. Santiago de Chile: CEDETI UC.

Valenzuela, J.P., Bellei, C. y Ríos, D.D.L. (2014). Socioeconomic school segregation in a marketoriented educational system. The case of Chile. Journal of Education Policy, 29, 217-241. http://dx.doi.org/10.1080/02680939.2013.80 6995

Van der Ven, S., Kroesbergen, E., Boom, J. y 
Leseman, P. (2013). The structure of executive functions in children: A closer examination of inhibition, shifting, and updating. British Journal of Developmental Psychology, 31(1), 70-87. http://dx.doi.org/10.1111/j.2044835X.2012.02079.x

Vygotsky, L. (1978). Mind in Society: The Development of Higher Psychological Processes. London: Harvard University Press.

Wiebe, S., Sheffield, T., Nelson, J., Clark, C., Chevalier, N. y Espy, K. (2011). The struc- ture of executive function in 3-year-olds. Journal of Experimental Child Psychology, 108(3), 436-452. http://dx.doi.org/10.1016/j. jecp.2010.08.008

Zelazo, P. y Müller, U. (2002). Executive function in typical and atypical development. En U. Goswami (Ed.), Handbook of childhood cognitive development (pp.445-469). Oxford, UK: Blackwell. http://dx.doi. org/10.1002/9780470996652.ch20

Recibido: 8 de marzo de 2018 Aceptado: 7 de enero de 2019 